\title{
A Comparative Study on Root Canal Repair Materials: A Cytocompatibility Assessment in L929 and MG63 Cells
}

\author{
Yuqing Jiang, Qinghua Zheng, Xuedong Zhou, Yuan Gao, and Dingming Huang \\ State Key Laboratory of Oral Diseases Department of Conservative Dentistry and Endodontics, West China Hospital of Stomatology, \\ Sichuan University, Chengdu 610041, China
}

Correspondence should be addressed to Dingming Huang; dingminghuang@163.com

Received 24 August 2013; Accepted 20 October 2013; Published 12 January 2014

Academic Editors: R. M. Love and S. Malkoç

Copyright (C) 2014 Yuqing Jiang et al. This is an open access article distributed under the Creative Commons Attribution License, which permits unrestricted use, distribution, and reproduction in any medium, provided the original work is properly cited.

Cytocompatibility of repair materials plays a significant role in the success of root canal repair. We conducted a comparative study on the cytocompatibility among iRoot BP Plus, iRoot FS, ProRoot MTA, and Super-EBA in L929 cells and MG63 cells. The results revealed that iRoot FS was able to completely solidify within 1 hour. iRoot BP Plus required 7-day incubation, which was much longer than expected (2 hours), to completely set. ProRoot MTA and Super-EBA exhibited a similar setting duration of 12 hours. All the materials except Super-EBA possessed negligible in vitro cytotoxicity. iRoot FS had the best cell adhesion capacity in both L929 and MG63 cells. With rapid setting, negligible cytotoxicity, and enhanced cell adhesion capacity, iRoot FS demonstrated great potential in clinical applications. Future work should focus on longer-term in vitro cytocompatibility and an in vivo assessment.

\section{Introduction}

The selection of the repair material is critical to perform a successful apical root-end surgery or root perforation repair. As a root repair materials, the materials should have excellent characteristics including acceptable biocompatibility, stability in physical and chemical property, radiopacity, set in a wet environment, and good sealing capability. In addition to this traditional concept of the purpose, it has recently been put forward that a root repair material should be able to actively stimulate tissue regeneration, especially after surgical procedures or apical pathosis. The relevant materials should be osteoconductive or osteoinductive [1-3]. So the cytocompatibility of the repair materials plays a significant role in the success of root canal repair [4].

A colorimetric [3-(4,5-dimethyl-thyazol-2-yl)-2,5-diphenyltetrazolium bromide] (MTT) assay is able to determine cellular viability based on the production of a colored formazan compound $[5,6]$, and this assay kit has been well documented to be a simple and reliable method for the in vitro cytotoxicity evaluation of different root canal repair materials [7]. A number of fibroblast cell lines, including L929 and 3T3, have been widely used for MTT assays due to their availability and reproducible outcomes
[8-10]. Since there is direct contact between root canal repair materials and periapical tissues, these materials are expected to exhibit osteoinductive or osteoconductive properties that promote bone deposition and eventually root canal repair $[4,11]$. Thus, it is also important to assess the cytocompatibility of root canal repair materials on osteoblast-like cells [12].

Various repair materials have been developed for root canal repair. Among these, Super-EBA and mineral trioxide aggregate (MTA) are the most commonly used materials in clinical applications [13-15], though some limitations exist in practice. For example, Super-EBA exhibits cytotoxicity due to the leaching of free eugenol [16-19]. In another variation, MTA is tissue-benign, but the long setting time and the difficulty to maintain the consistency of the material still remain issues $[4,20,21]$.

Recently, a series of iRoot materials (iRoot BP Plus and iRoot FS) have emerged as a new generation of root canal repair materials that have a shorter setting duration. These materials are bioceramic-based and the main compositions include calcium silicates and monobasic calcium phosphate, which facilitates the cytocompatibility of these materials [22]. Moreover, previous studies have demonstrated that the contact between osteoblasts and bioceramic components 
enhances the production of cytokines such as interleukins and tumor necrosis factor [23]. The elevated expression of these bone-resorptive cytokines has a beneficial effect on bone formation [24]. Thus, these materials show high potential for root canal repair.

However, to the best of our knowledge, very few studies have been reported regarding the cytocompatibility and cellmaterial interaction of these materials. Therefore, the aim of this study was to conduct a comparative assessment on the surface morphology and the cell adhesion capacity of iRoot BP Plus, iRoot FS, ProRoot MTA, and Super-EBA on both fibroblast and osteoblast-like cells models. Furthermore, the time-course in vitro cytotoxicity of these materials was assessed.

\section{Materials and Methods}

2.1. Materials. The culture medium prepared was Dulbecco's modified Eagle medium (DMEM, Hyclone) supplemented with $10 \%$ fetal bovine serum (FBS, Hyclone) and antibiotics (Penicillin $100 \mathrm{U} / \mathrm{mL}$ and Streptomycin $100 \mu \mathrm{g} / \mathrm{mL}$, Gibco). The osteoblast-like cells (MG63) and mouse fibroblast cells (L929) were supplied from State Key Laboratory of Oral Diseases, Sichuan University, China. iRoot BP Plus and iRoot FS were supplied from Innovative Bioceramix Inc. ProRoot MTA was supplied from Dentsply Tulsa Dental. Super-EBA was purchased from Bosworth Co. MTT was purchased from Sigma.

2.2. Specimen Preparation. All repair materials (iRoot BP Plus, iRoot FS, ProRoot MTA, and Super-EBA) were prepared under aseptic conditions. iRoot BP Plus and iRoot FS were premixed and packaged in paste forms. ProRoot MTA (in powder form) was mixed with distilled water and SuperEBA was mixed with the working solution supplied by the manufacturer. These materials were placed in sterile custom-made Teflon cylindrical molds $(10 \mathrm{~mm}$ diameter and $3 \mathrm{~mm}$ thickness) at $37^{\circ} \mathrm{C}$ under $100 \%$ humidity. iRoot FS has hardened with a $500 \mathrm{~g}$ load by Knoop Hardness Tester (Wilson Instruments, Norwood, MA) after being in the mold for 1 hour. ProRoot MTA and Super-EBA exhibited a similar setting duration of 12 hours. iRoot BP Plus was completely solidified after 7 days.

All the samples were then covered with gauze and immersed in distilled water for solidification at $37^{\circ} \mathrm{C}$ under $100 \%$ humidity for 7 days. The solid materials were subsequently incubated in DMEM following a 1-hour ultraviolet light exposure at $37^{\circ} \mathrm{C}$ with $5 \% \mathrm{CO}_{2}$.

2.3. Cytotoxicity Assay. The cytotoxicities of these materials were determined as previously described [2, 22]. After 24hour incubation in DMEM, elutes of each sample (with a surface area to volume ratio of $250 \mathrm{~mm}^{2} / \mathrm{mL}$ ) [25] at different time intervals (1, 3, 7, and 14 days) were extracted and filtered through a $0.22 \mu \mathrm{m}$ filter (Millipore) to remove particulate impurities. These elutes along with their dilutions in DMEM (50\% and 25\%, resp., without FBS) were subsequently used for cell culture. Fresh DMEM was examined as a control.
Cell suspensions (100 $\mu \mathrm{L} /$ well) were transferred into 96well plates at a concentration of $5 \times 10^{4}$ cells/well and incubated for 24 hours. Then, the cells were removed and the elutes of different materials $(200 \mu \mathrm{L})$ were added for another 24-hour incubation period. The relative quantities of cells (optical density (OD) at $490 \mathrm{~nm}$ ) were evaluated by using a colorimetric (MTT) assay on a microplate reader (Bio-Rad). The relative cell viability was expressed as the ratio of the OD value of elutes at each condition (original elutes and their dilutions) over the control (DMEM).

2.4. Surface Morphology and Element Analysis. Each sample was pre-incubated in phosphate buffered saline (PBS) for 2 weeks and the media was refreshed every day. The surface morphology of the prepared samples was examined by using a scanning electron microscope (SEM, Hitachi, S-3000N). The element analysis was performed by a built-in energy dispersive X-ray spectroscope (EDX).

2.5. In Vitro Cell Adhesion. Each sample was preincubated in PBS for 2 weeks and the medium was refreshed every day. Cells were loaded onto the samples at a concentration of 5 $\times 10^{4}$ cells/sample and allowed to adhere for 24 hours. After incubation, the cell-adhered samples were washed with PBS three times gently and fixed by $2.5 \%$ glutaraldehyde for 4 hours. The fixed samples were then treated with a series of graded ethanol solutions $(30 \%, 50 \%, 70 \%, 80 \%, 90 \%, 95 \%$, and 100\%, 15 min each) and then examined by SEM.

\section{Results}

3.1. In Vitro Cytotoxicity. The in vitro cytotoxicities of these materials were compared using the MG63 model (Figure 1) and the L929 model (Figure 2), respectively. The results in the MG63 system were similar to those in the L929 system. In both systems, elutes from the Super-EBA 7-day treatment exhibited relative viabilities less than $60 \%$. The relative viability was dramatically enhanced $(90 \%)$ by a 14 day culture. Serial dilutions of these elutes had no statistically significant effect on the relative viability. On the other hand, the relative viabilities at different incubation durations (up to 14 days) and different dilutions were all around 95\% in the iRoot BP Plus, iRoot FS, and ProRoot MTA groups. These were significantly higher than those of the Super-EBA group.

3.2. Surface Morphology and Element Analysis. The surface morphologies of the materials are illustrated in Figures 3(a)-3(d). iRoot BP Plus and iRoot FS possessed a similar morphology. Schistose and flaky crystals in varied sizes were observed (Figures 3(a) and 3(b)). The average length of flakes was $20 \mu \mathrm{m}$ for iRoot BP Plus and $5 \mu \mathrm{m}$ for iRoot FS, respectively. ProRoot MTA showed hexagonalshaped granules with an average diameter of around $5 \mu \mathrm{m}$ (Figure 3(c)). The EDX analysis revealed that iRoot BP Plus, iRoot FS, and ProRoot MTA possessed similar element compositions (calcium, carbon, oxygen, and phosphorus). Super-EBA showed a poorly crystallized morphology with large dendrites ( $100 \mu \mathrm{m}$ length), and the main compositions 


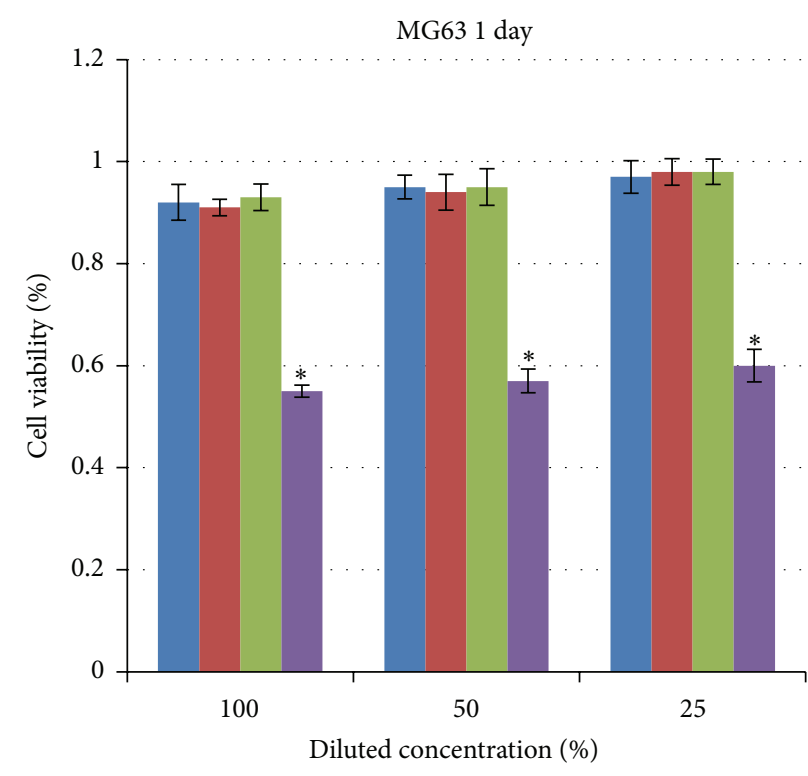

(a)

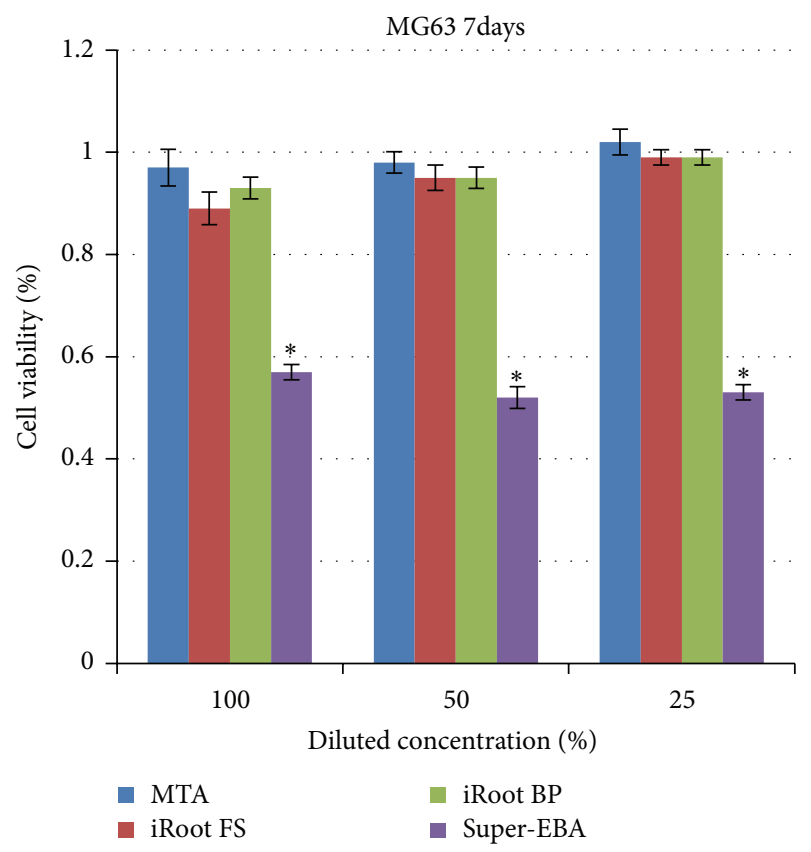

(c)

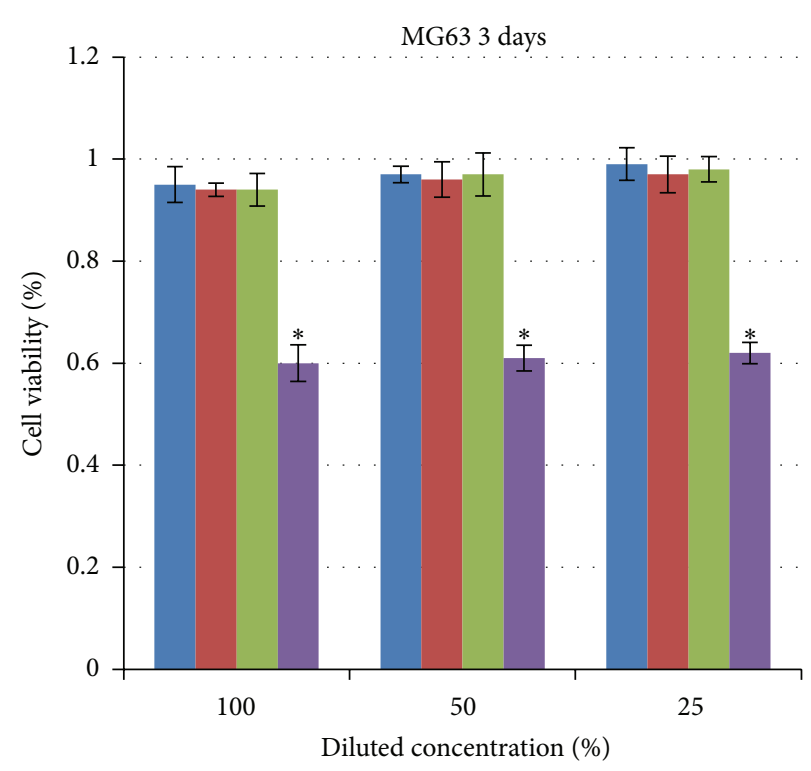

(b)

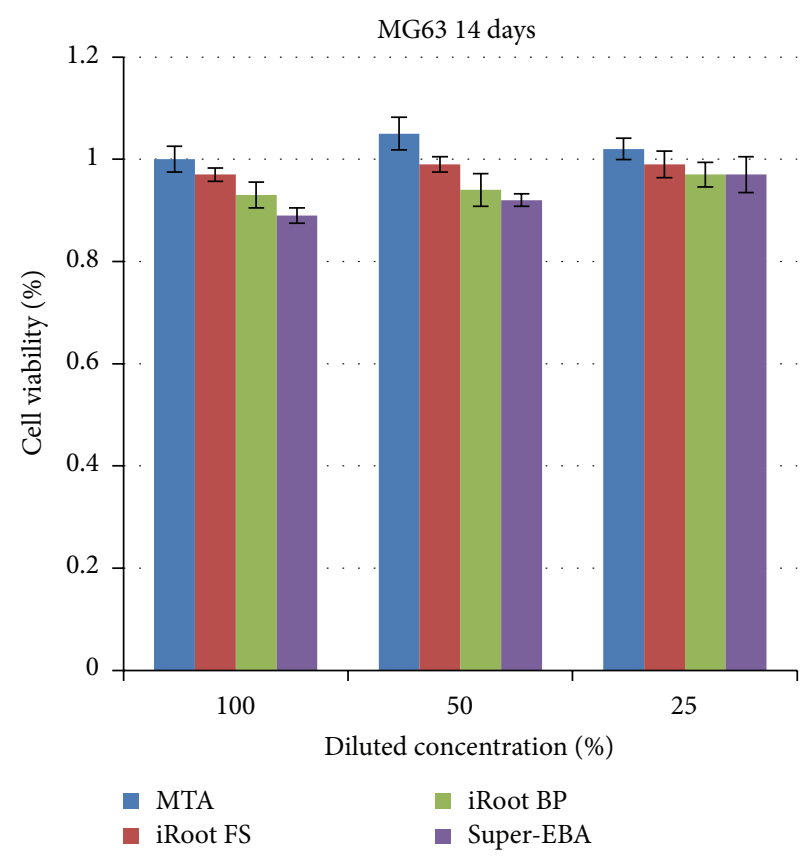

(d)

FIGURE 1: Relative cell viability of different materials determined by MTT assay in MG63 cells upon 1-day (a), 3-day (b), 7-day (c), and 14-day (d) incubation, respectively. ${ }^{*} P<0.05$.

included zinc, aluminum carbon, oxygen, and phosphorus (Figure 3(h)).

3.3. Cell Adhesion. As shown in Figure 4, MG63 cells were able to adhere and spread on all the specimens with a characteristic polygonal shape. The iRoot FS group had the highest adhesion density and the highest presence of filopodia (Figure 4(b)) when compared with other specimens.

The adhesion of L929 cells on the specimens is illustrated in Figure 5. After 24-hour incubation, cells on iRoot BP Plus are almost circular, indicating slower attachment and less spread. The cells on the other specimens exhibited a spindlelike shape, which is typical for fibroblasts. The adhesion density in the iRoot FS group was higher than that on other specimens.

\section{Discussion}

iRoot series materials are described as bioceramic materials developed for permanent root canal repair applications with 


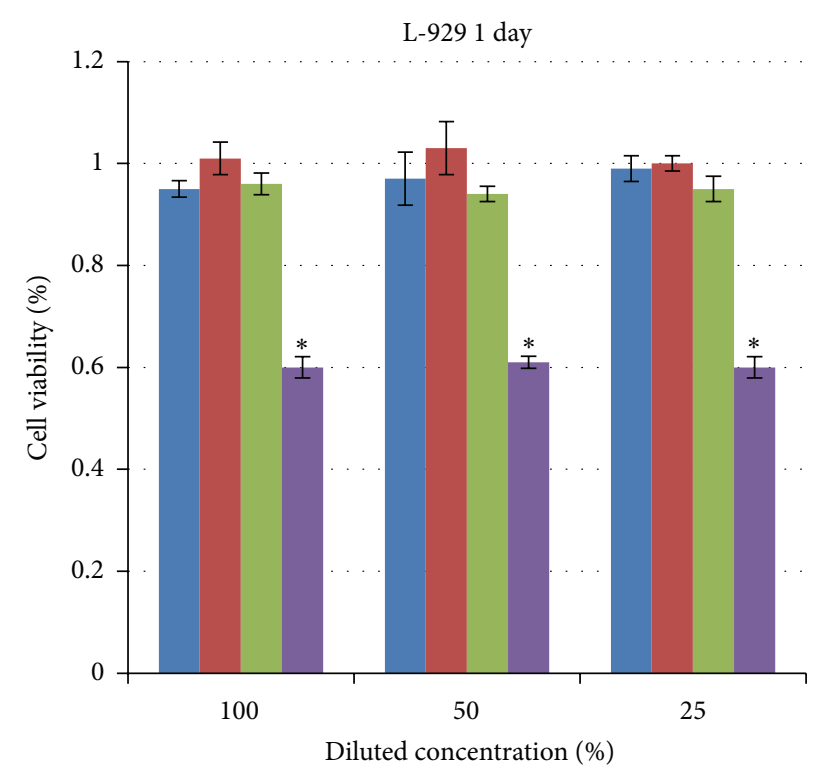

(a)

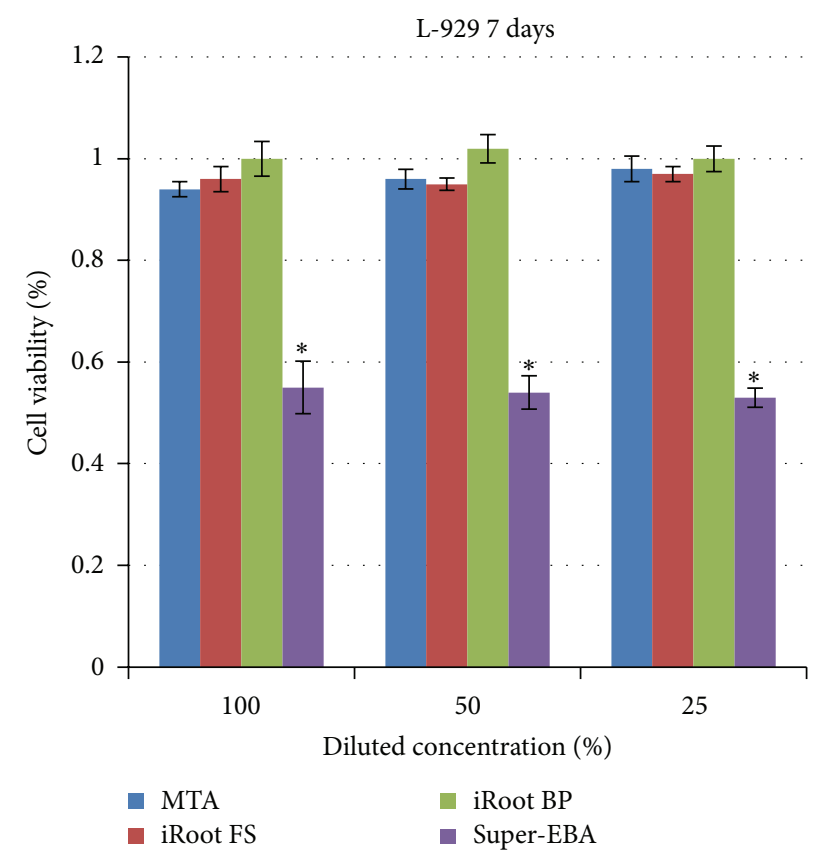

(c)

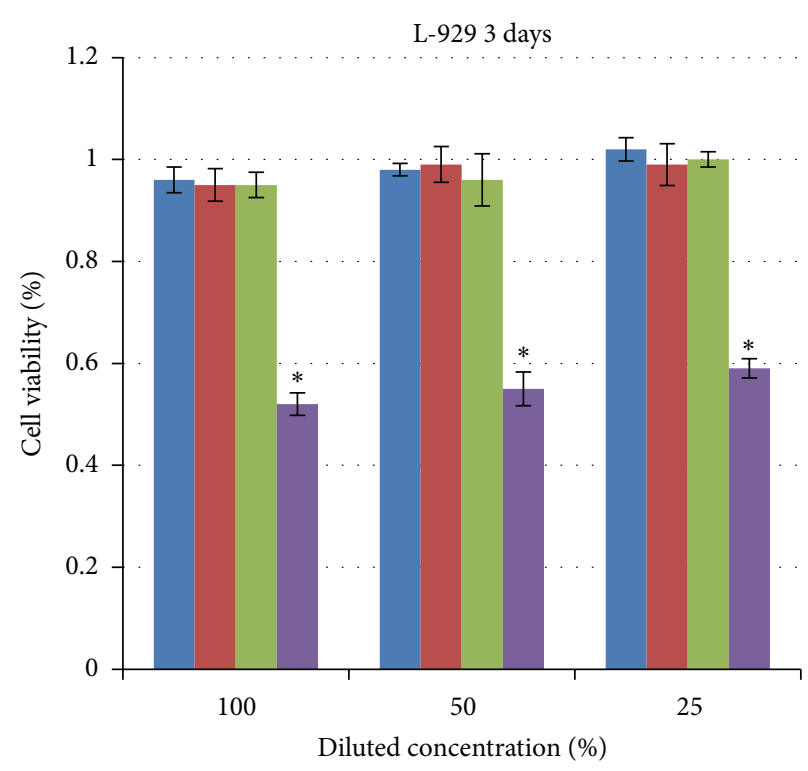

(b)

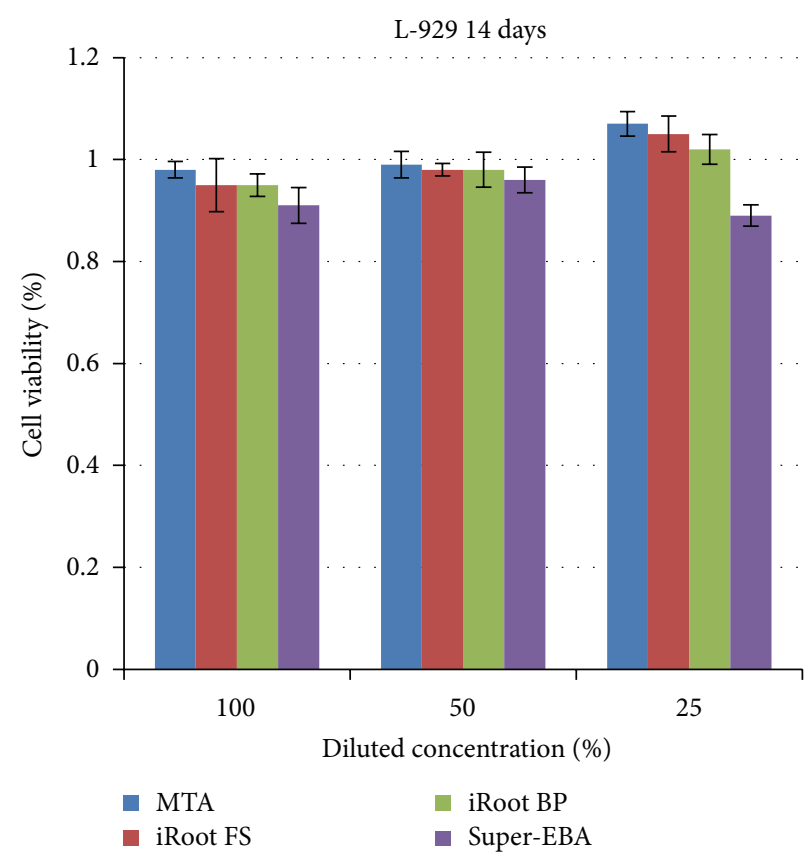

(d)

FIGURE 2: Relative cell viability of different materials determined by MTT assay in L929 cells upon 1-day (a), 3-day (b), 7-day (c), and 14-day (d) incubation, respectively. ${ }^{*} P<0.05$.

improved handling properties and shorter setting times. They are ready-to-use white hydraulic premixed putty and are composed of calcium silicates, zirconium oxide, tantalum pentoxide, calcium phosphate monobasic, and filler agents. In addition, the manufacturer claims that there is no shrinking during the setting period and that the material is insoluble, radiopaque, and aluminum-free.

The ultimate goal of root canal repair procedure is to permanently seal infected or damaged root canals and to promote the healing of the repair area $[1,26]$. For this purpose, a number of materials have been developed and widely used in clinics. In this study, we evaluated four marketavailable repair materials (iRoot BP Plus, iRoot FS, ProRoot MTA, and Super-EBA). One important criterion of these materials is the setting time $[7,27]$. A reduction in setting duration has a beneficial effect on patient relief and reducing bacterial infection [5]. In this study, iRoot FS material was able to completely solidify within one hour at $37^{\circ} \mathrm{C}$ in $100 \%$ relative humidity given an extra load $(500 \mathrm{~g})$ to harden the material. Meanwhile, iRoot BP Plus formed a stable structure 
iRoot BP Plus

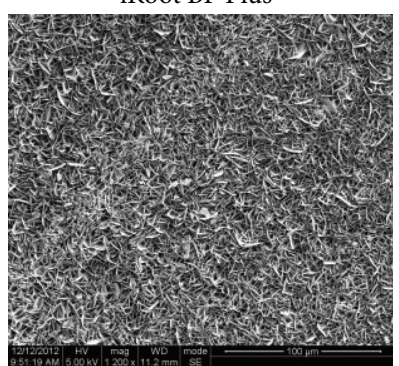

(a)

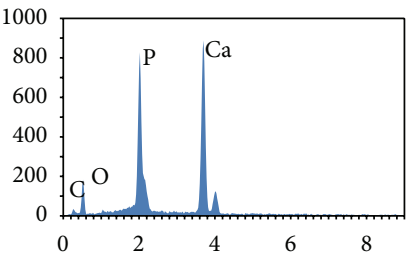

(e)
iRoot FS

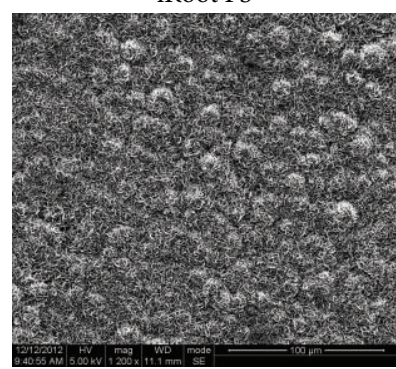

(b)

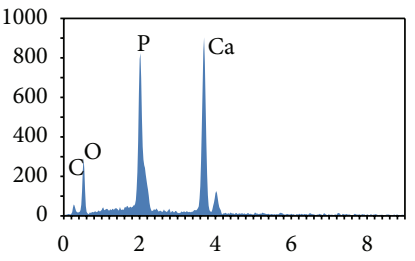

(f)

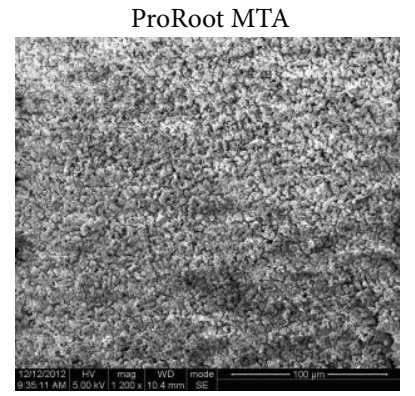

(c)

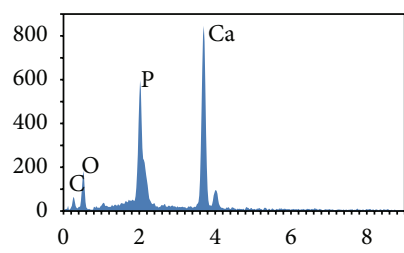

(g)

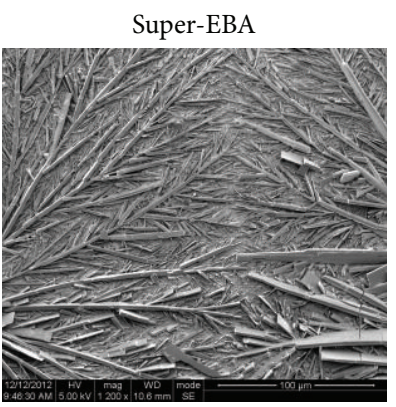

(d)

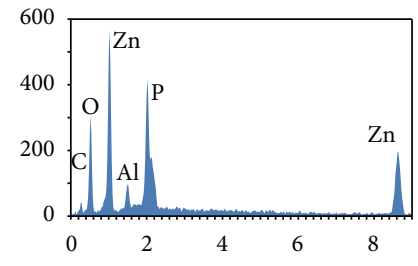

(h)

FIGURE 3: SEM images and corresponding EDX spectrum of iRoot BP Plus ((a) and (e)), iRoot FS ((b) and (f)), ProRoot MTA ((c) and (g)), and Super-EBA $((\mathrm{d})$ and $(\mathrm{h}))$. The scale bars shown are $100 \mu \mathrm{m}$.

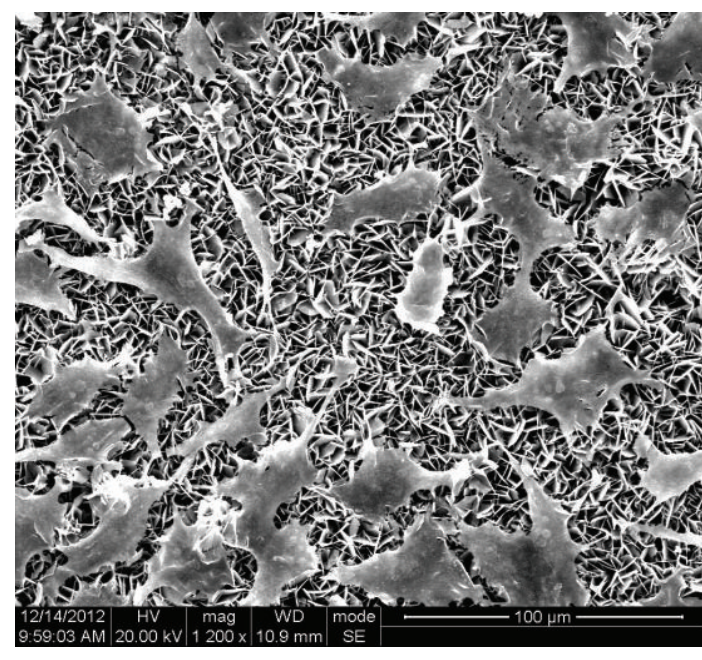

(a)

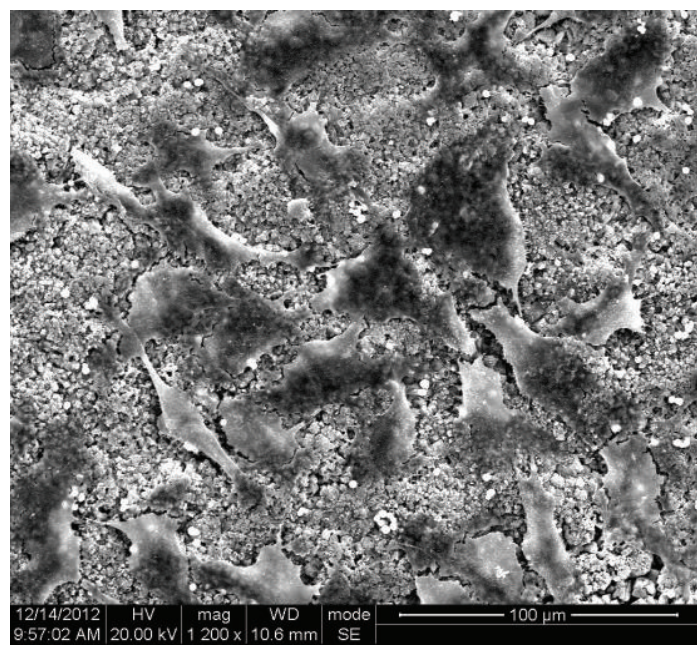

(c)

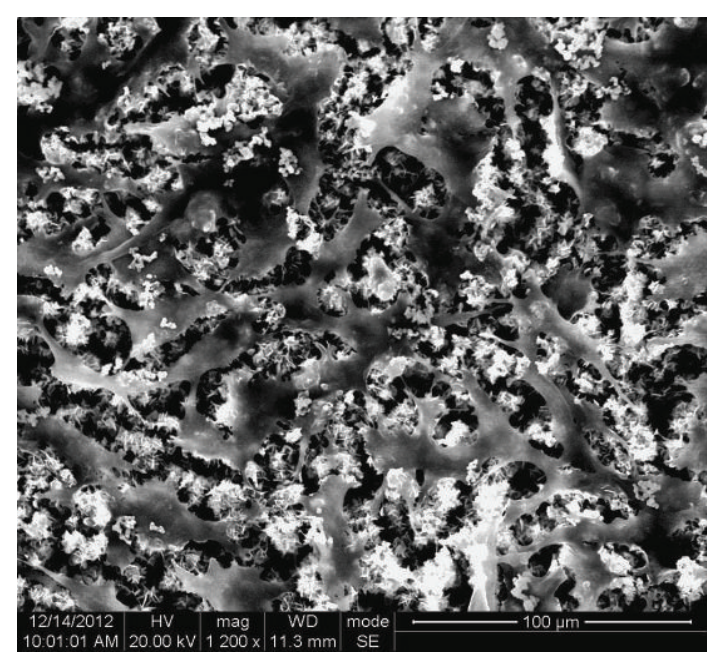

(b)

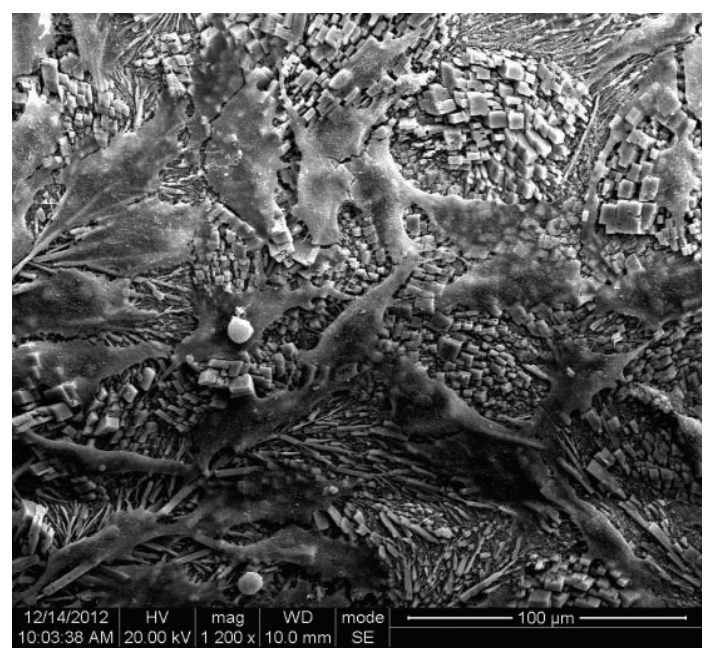

(d)

Figure 4: SEM images of iRoot BP Plus (a), iRoot FS (b), ProRoot MTA (c), and Super-EBA (d) after MG63 cell adhesion. The scale bars shown are $100 \mu \mathrm{m}$. 


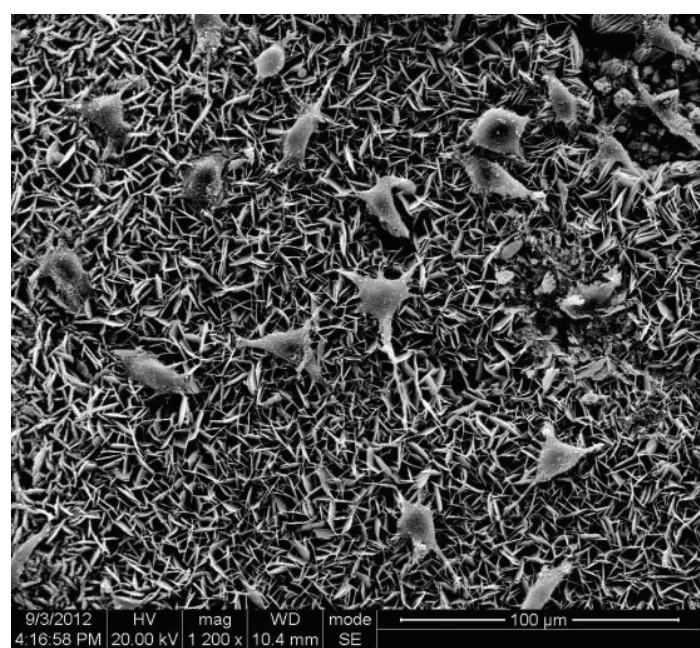

(a)

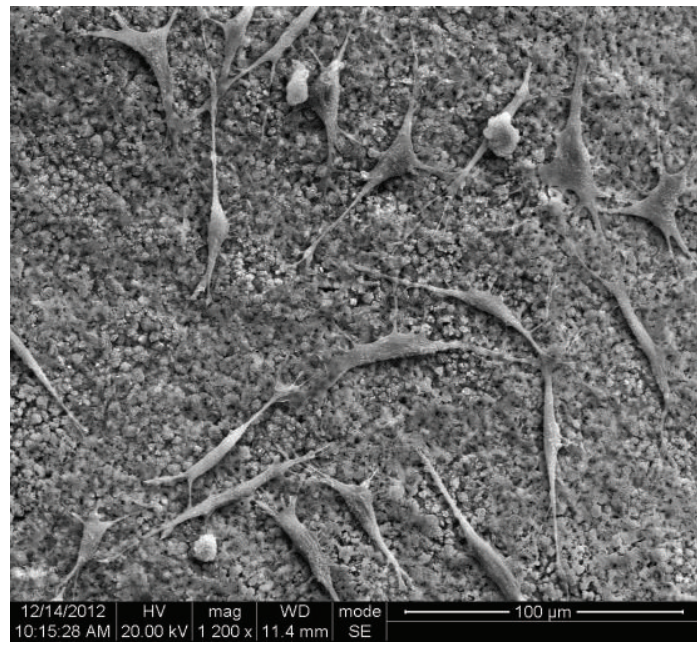

(c)

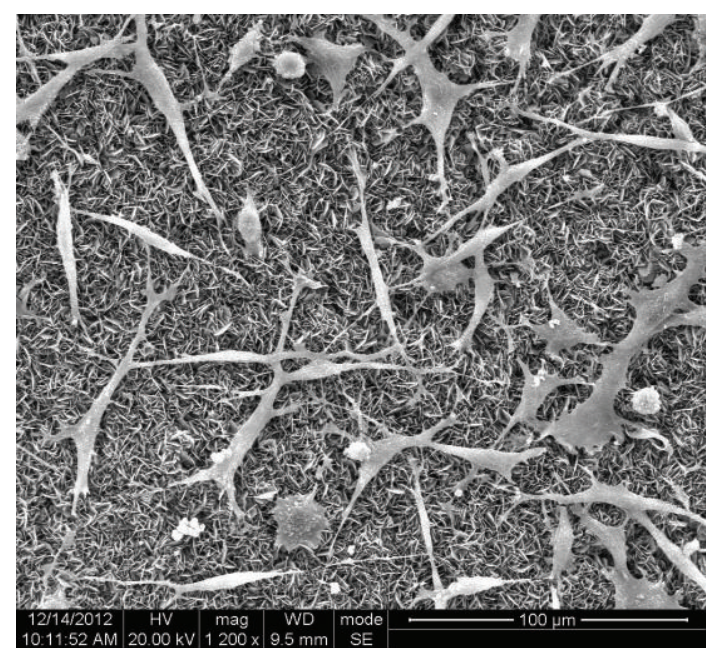

(b)

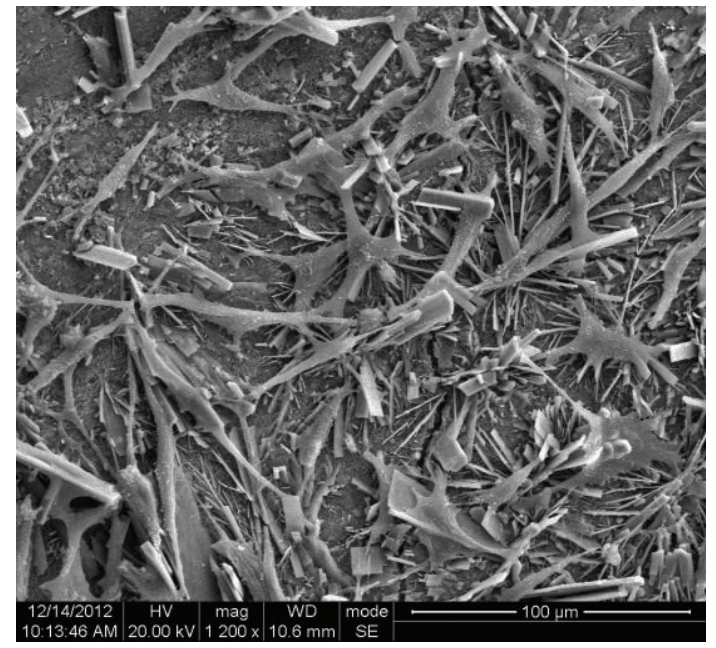

(d)

FIGURE 5: SEM images of iRoot BP Plus (a), iRoot FS (b), ProRoot MTA (c), and Super-EBA (d) after L929 cell adhesion. The scale bars shown are $100 \mu \mathrm{m}$.

following seven days of incubation. This was in contrast with the manufacturer's instruction, which indicated that the mixture could be fixed within around 2 hours. A recent study also observed a similar slower setting process (at least 5 days) of iRoot BP Plus [28]. The reason for the delayed setting is still under investigation, but we anticipate that it might be attributed to the humidity-sensitive properties of the compositions. Finally, both ProRoot MTA and SuperEBA required a setting time of at least 12 hours.

The success of root canal therapy relies on the cytocompatibility of the repair materials [22]. In this study, we first examined the cell adhesion capacity of these materials, and the results revealed that iRoot FS exhibited the best cell adhesion (on both L929 and MG63 models) among all the materials. Previous studies demonstrated that cell adhesion was highly dependent on the surface morphology and topography of the materials $[29,30]$. Berry et al. indicated that a finer microarchitecture resulted in higher cell attachment and subsequently proliferation rate [29]. These data were in agreement with the results from our current study. Indeed, iRoot BP Plus and iRoot FS possessed a similar microstructure on the surface, with different particle (flake) sizes. More cells (both L929 and MG63) were attached on materials with smaller particle sizes (iRoot FS).

Moreover, in this study, the in vitro cytotoxicities of these materials were evaluated by using an MTT assay. The cytotoxicity of the materials could be caused by the presence of toxic components or soluble materials that leach into the surrounding fluids in the bony crypt [17]. Our results revealed that Super-EBA exhibited a significantly higher cytotoxicity than the other materials upon in vitro culture. Although Super-EBA has been widely used in clinical practice, the cytotoxicity remains an issue, and recent studies have demonstrated that the cytotoxicity is mainly induced by the leaching of free eugenol [13]. On the other hand, in this study, iRoot BP plus, iRoot FS, and ProRoot MTA 
TABLE 1: Characteristics of different root canal repair materials.

\begin{tabular}{lcclc}
\hline Specimen & Initial form & Setting time & Surface morphology & Particle size \\
\hline iRoot BP Plus & Premixed paste & 7 days ${ }^{*}$ & Schistose and flaky crystals & $20 \mu \mathrm{m}$ \\
iRoot FS & Premixed paste & 1 hour & Schistose and flaky crystals & $5 \mu \mathrm{m}$ \\
ProRoot MTA & Powder & 4 hours & Hexagonal granules & $5 \mu \mathrm{m}$ \\
Super-EBA & Powder & 12 hours & Dendrites & $100 \mu \mathrm{m}$ \\
\hline
\end{tabular}

${ }^{*}$ Note: the expected setting time of iRoot BP Plus is around 2 hours according to manufacturer's instruction.

exhibited negligible cytotoxicity. This can be explained by the nontoxic components, including calcium and phosphorus, of these materials. Furthermore, the presence of bioceramic facilitated the formation of a hydroxyapatite or apatitelike layer (biomineralization), which further stabilizes the structure and prevents the overdose of component leaching [31-33]. These results are in agreement with previous studies [22].

Taken together, iRoot FS demonstrated great potential in further clinical applications due to its rapid setting, negligible cytotoxicity, and enhanced cell adhesion capacity compared with other commonly used root canal repair materials (Table 1).

\section{Conclusion}

A comparative study was conducted on four root canal repair materials by using both L929 and MG63 cells. The results demonstrated that iRoot FS exhibited the best cell adhesion capacity, and only Super-EBA possessed in vitro cytotoxicity. Given the rapid solidification (within one hour) of iRoot FS, this material showed high potential for further clinical applications. Future work should focus on long-term in vitro cytotoxicity and an in vivo assessment.

\section{Conflict of Interests}

The authors declare that there is no conflict of interests regarding the publication of this paper.

\section{Acknowledgments}

The study was supported by the National Natural Science foundation of China (Grant nos. 81070827 and 11272226) and The Science and Technology support program of Sichuan province (no. 2011SZ0031).

\section{References}

[1] G. Bergenholtz, P. Hørsted-Bindslev, and C. Reit, Textbook of Edodontology, vol. 193, Blackwell Publishing Company, Munksgaard, Denmark, 2010.

[2] P. Xu, J. Liang, G. Dong, L. Zheng, and L. Ye, "Cytotoxicity of RealSeal on human osteoblast-like MG63 cells," Journal of Endodontics, vol. 36, no. 1, pp. 40-44, 2010.

[3] S. Desai and N. Chandler, "Calcium hydroxide-based root canal sealers: a review," Journal of Endodontics, vol. 35, no. 4, pp. 475480, 2009.
[4] J. Ma, Y. Shen, S. Stojicic, and M. Haapasalo, "Biocompatibility of two novel root repair materials," Journal of Endodontics, vol. 37, no. 6, pp. 793-798, 2011.

[5] A. Z. AlAnezi, J. Jiang, K. E. Safavi, L. S. W. Spangberg, and Q. Zhu, "Cytotoxicity evaluation of endosequence root repair material," Oral Surgery, Oral Medicine, Oral Pathology, Oral Radiology and Endodontology, vol. 109, no. 3, pp. e122-e125, 2010.

[6] W. Zhang, Z. Li, and B. Peng, "Ex vivo cytotoxicity of a new calcium silicate-based canal filling material," International Endodontic Journal, vol. 43, no. 9, pp. 769-774, 2010.

[7] B. A. Damas, M. A. Wheater, J. S. Bringas, and M. M. Hoen, "Cytotoxicity comparison of mineral trioxide aggregates and endosequence bioceramic root repair materials," Journal of Endodontics, vol. 37, no. 3, pp. 372-375, 2011.

[8] S. Bouillaguet, J. C. Wataha, F. R. Tay, M. G. Brackett, and P. E. Lockwood, "Initial In vitro biological response to contemporary endodontic sealers," Journal of Endodontics, vol. 32, no. 10, pp. 989-992, 2006.

[9] W. Geurtsen, W. Spahl, and G. Leyhausen, "Residual monomer/ additive release and variability in cytotoxicity of light-curing glass-ionomer cements and compomers," Journal of Dental Research, vol. 77, no. 12, pp. 2012-2019, 1998.

[10] Z. Yilmaz, A. L. Dogan, O. Ozdemir, and A. Serper, "Evaluation of the cytotoxicity of different root canal sealers on L929 cell line by MTT assay," Dental Materials Journal, vol. 31, no. 6, pp. 1028-1032, 2012.

[11] B. Sağsen, Y. Ustün, K. Pala, and S. Demırbuğa, "Resistance to fracture of roots filled with different sealers," Dental Materials Journal, vol. 31, no. 4, pp. 528-532, 2012.

[12] S. Bonson, B. G. Jeansonne, and T. E. Lallier, "Root-end filling materials alter fibroblast differentiation," Journal of Dental Research, vol. 83, no. 5, pp. 408-413, 2004.

[13] A. Samara, Y. Sarri, D. Stravopodis, G. N. Tzanetakis, E. G. Kontakiotis, and E. Anastasiadou, "A comparative study of the effects of three root-end filling materials on proliferation and adherence of human periodontal ligament fibroblasts," Journal of Endodontics, vol. 37, no. 6, pp. 865-870, 2011.

[14] E. Bodrumlu, "Biocompatibility of retrograde root filling materials: a review," Australian Endodontic Journal, vol. 34, no. 1, pp. 30-35, 2008.

[15] S. Friedman, "Retrograde approaches in endodontic therapy," Endodontics \& Dental Traumatology, vol. 7, no. 3, pp. 97-107, 1991.

[16] M. Asrari and D. Lobner, "In vitro neurotoxic evaluation of rootend-filling materials," Journal of Endodontics, vol. 29, no. 11, pp. 743-746, 2003.

[17] K. Keiser, C. Chad Johnson, and D. A. Tipton, "Cytotoxicity of mineral trioxide aggregate using human periodontal ligament fibroblasts," Journal of Endodontics, vol. 26, no. 5, pp. 288-291, 2000 
[18] O. R. Al-Sa'eed, A. S. Al-Hiyasat, and H. Darmani, “The effects of six root-end filling materials and their leachable components on cell viability," Journal of Endodontics, vol. 34, no. 11, pp. 14101414, 2008.

[19] C.-P. Lin, Y.-J. Chen, Y.-L. Lee et al., "Effects of root-end filling materials and eugenol on mitochondrial dehydrogenase activity and cytotoxicity to human periodontal ligament fibroblasts," Journal of Biomedical Materials Research B, vol. 71, no. 2, pp. 429-440, 2004.

[20] H. W. Roberts, J. M. Toth, D. W. Berzins, and D. G. Charlton, "Mineral trioxide aggregate material use in endodontic treatment: a review of the literature," Dental Materials, vol. 24, no. 2, pp. 149-164, 2008.

[21] M. Parirokh and M. Torabinejad, "Mineral trioxide aggregate: a comprehensive literature review-part III: clinical applications, drawbacks, and mechanism of action," Journal of Endodontics, vol. 36, no. 3, pp. 400-413, 2010.

[22] G. De-Deus, A. Canabarro, G. G. Alves, J. R. Marins, A. B. R. Linhares, and J. M. Granjeiro, "Cytocompatibility of the ready-to-use bioceramic putty repair cement iRoot BP Plus with primary human osteoblasts," International Endodontic Journal, vol. 45, no. 6, pp. 508-513, 2012.

[23] M. Ciasca, A. Aminoshariae, G. Jin, T. Montagnese, and A. Mickel, "A comparison of the cytotoxicity and proinflammatory cytokine production of EndoSequence root repair material and ProRoot mineral trioxide aggregate in human osteoblast cell culture using reverse-transcriptase polymerase chain reaction," Journal of Endodontics, vol. 38, no. 4, pp. 486-489, 2012.

[24] F. de Paula and C. J. Rosen, "Bone remodeling and energy metabolism: new perspectives," Bone Research, vol. 1, no. 1, pp. 72-84, 2013.

[25] ISO 10993-5: 2009 Biological evaluation of medical devices part 5: tests for in vitro cytotoxicity.

[26] I. Willershausen, A. Callaway, B. Briseño, and B. Willershausen, "In vitro analysis of the cytotoxicity and the antimicrobial effect of four endodontic sealers," Head and Face Medicine, vol. 7, no. 1, article 15, 2011.

[27] J.-S. Song, F. K. Mante, W. J. Romanow, and S. Kim, "Chemical analysis of powder and set forms of Portland cement, gray ProRoot MTA, white ProRoot MTA, and gray MTA-Angelus," Oral Surgery, Oral Medicine, Oral Pathology, Oral Radiology and Endodontology, vol. 102, no. 6, pp. 809-815, 2006.

[28] M. R. Modareszadeh, P. M. di Fiore, D. A. Tipton, and N. Salamat, "Cytotoxicity and alkaline phosphatase activity evaluation of endosequence root repair material," Jounal of Endodontics, vol. 38, no. 8, pp. 1101-1105, 2012.

[29] C. C. Berry, G. Campbell, A. Spadiccino, M. Robertson, and A. S. G. Curtis, "The influence of microscale topography on fibroblast attachment and motility," Biomaterials, vol. 25, no. 26, pp. 5781-5788, 2004.

[30] C. Ji, N. Annabi, A. Khademhosseini, and F. Dehghani, "Fabrication of porous chitosan scaffolds for soft tissue engineering using dense gas $\mathrm{CO}_{2}$," Acta Biomaterialia, vol. 7, no. 4, pp. 16531664, 2011.

[31] N. K. Sarkar, R. Caicedo, P. Ritwik, R. Moiseyeva, and I. Kawashima, "Physicochemical basis of the biologic properties of mineral trioxide aggregate," Journal of Endodontics, vol. 31, no. 2, pp. 97-100, 2005.
[32] J. F. Reyes-Carmona, M. S. Felippe, and W. T. Felippe, "Biomineralization ability and interaction of mineral trioxide aggregate and white Portland cement with dentin in a phosphatecontaining fluid," Journal of Endodontics, vol. 35, no. 5, pp. 731736, 2009.

[33] F. R. Tay, D. H. Pashley, F. A. Rueggeberg, R. J. Loushine, and R. N. Weller, "Calcium phosphate phase transformation produced by the interaction of the Portland cement component of white mineral trioxide aggregate with a phosphate-containing fluid," Journal of Endodontics, vol. 33, no. 11, pp. 1347-1351, 2007. 


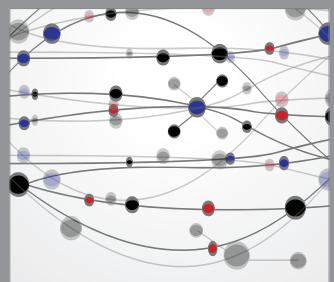

The Scientific World Journal
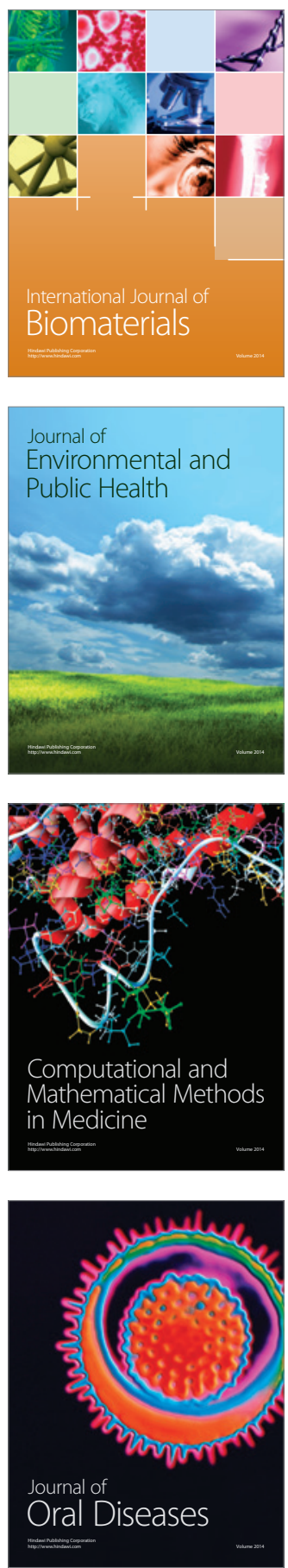
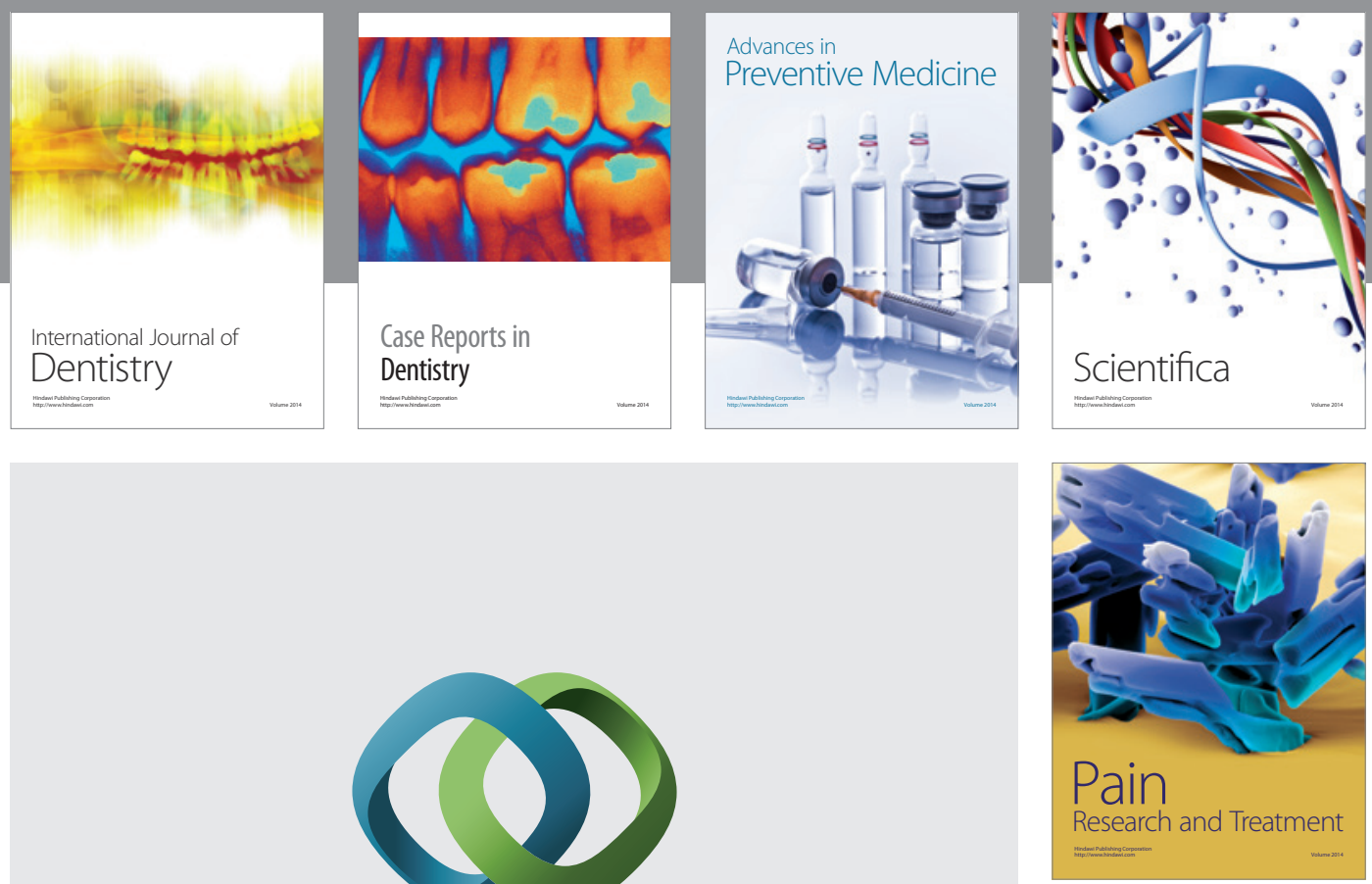

\section{Hindawi}

Submit your manuscripts at

http://www.hindawi.com
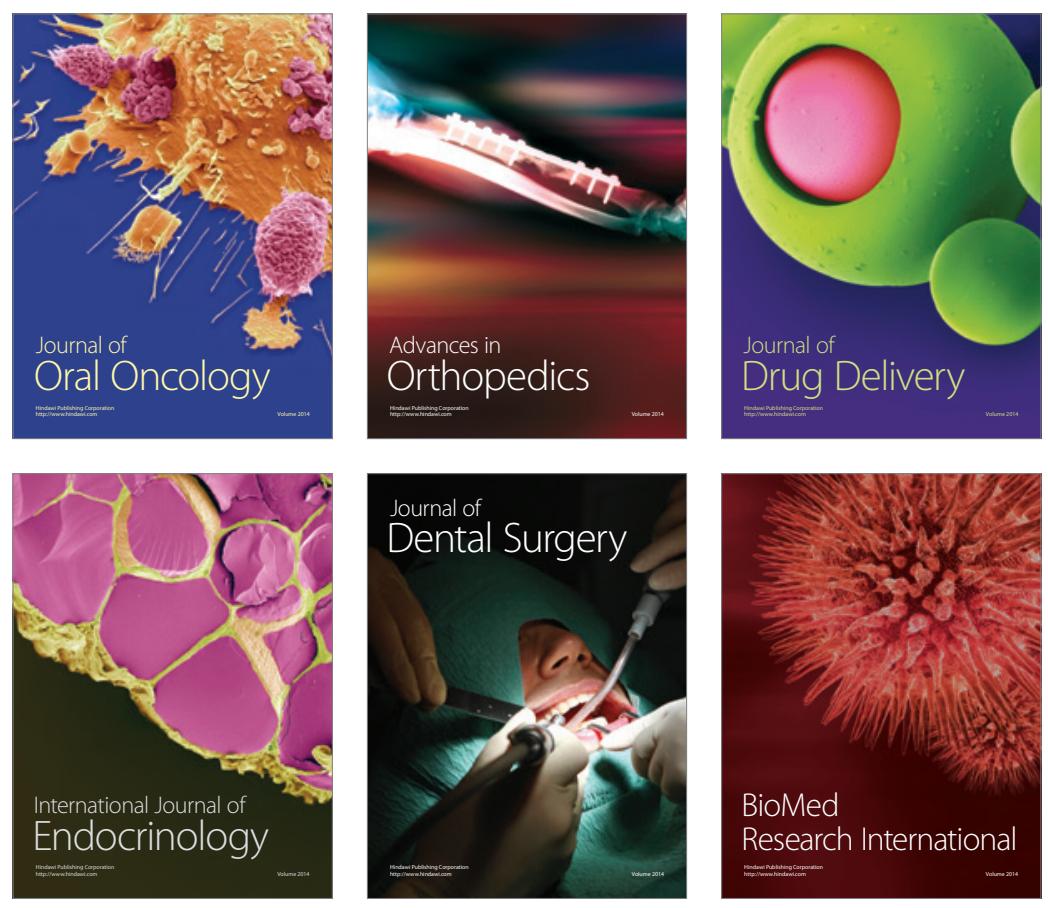

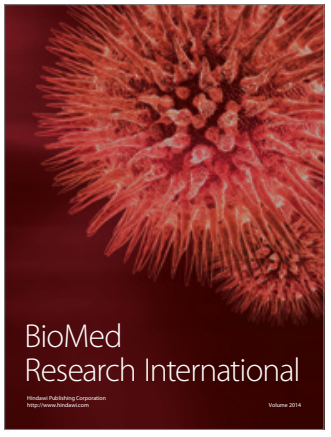

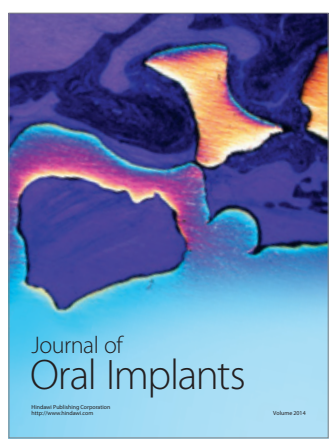
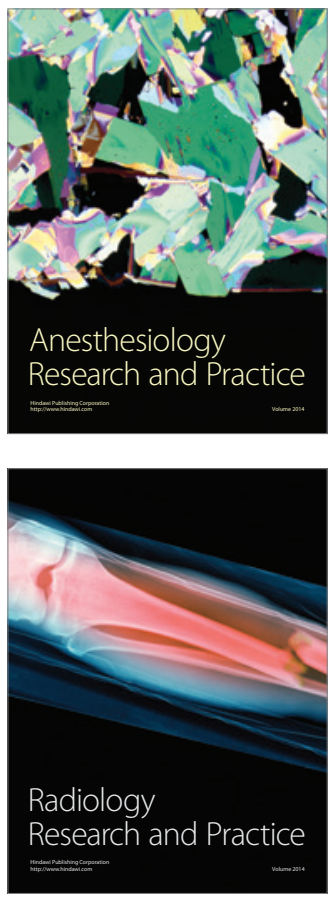